\title{
Identification of a novel function of Id-1 in mediating the anticancer responses of SAMC, a water-soluble garlic derivative, in human bladder cancer cells
}

\author{
HAO HU ${ }^{1,2^{*}}$, XIAO PENG ZHANG ${ }^{1 *}$, YU LIANG WANG ${ }^{1}$, CHEE WAI CHUA $^{2}$, SZE UE LUK $^{2}$, \\ YONG CHUAN WONG ${ }^{2}$, MING TAT LING ${ }^{2}$, XIAO FENG WANG ${ }^{1}$ and KE XIN XU ${ }^{1}$
}

\author{
${ }^{1}$ Department of Urology, Peking University People's Hospital, Peking University Health Science Center, Beijing; \\ ${ }^{2}$ Cancer Biology Group, Department of Anatomy, Faculty of Medicine, University of Hong Kong, Hong Kong SAR, P.R. China
}

Received June 9, 2010; Accepted October 4, 2010

DOI: $10.3892 / \mathrm{mmr} .2010 .380$

\begin{abstract}
Studies have shown that the expression of inhibitor of differentiation (Id-1) is increased in bladder cancer and is associated with drug resistance. S-allylmercaptocysteine (SAMC), a water-soluble component of garlic, is known to have a potent therapeutic effect on human cancer. The aim of this study was to investigate whether Id-1 expression mediates SAMC-induced cell death in bladder cancer cells. After generating stable Id-1-expressing and si-Id-1 transfectants in various bladder cancer cell lines, cell sensitivity to SAMC was compared by colony formation and MTT assays. The results indicated that Id-1 overexpression reduced the positive effect of SAMC on cell survival, while the inactivation of Id-1 increased cellular susceptibility to SAMC. Using DAPI staining, the apoptosis of bladder cancer cells induced by SAMC was shown to be negatively regulated by Id-1 expression. The expression of apoptosis-related proteins analyzed by Western blotting further supported the negative role of Id-1 in SAMC-induced apoptosis. Furthermore, by wound closure and type I collagen invasion assays, the inhibitory effect of SAMC on the invasion and migration of bladder cancer cells was found to be associated with the down-regulation of Id-1. Our results demonstrated that SAMC-induced apoptosis is associated with the Id-1 pathway, and that the inactivation of Id-1 enhances the ability of SAMC to inhibit the survival, invasion and migration of bladder cancer cells. These findings may lead to the development of novel therapeutic strategies for the treatment of bladder cancer.
\end{abstract}

Correspondence to: Dr Kexin Xu, Department of Urology, Peking University People's Hospital, 11 Xizhimennan Street, Xicheng, Beijing 100044, P.R. China

E-mail: xukexin@pkuph.edu.cn

*Contributed equally

Key words: inhibitor of differentiation, S-allylmercaptocysteine, bladder cancer cells

\section{Introduction}

Inhibitor of differentiation (Id-1), a member of the basic helixloop-helix (HLH) transcription factor family, lacks a basic domain for DNA binding. Id-1 therefore acts as a dominant inhibitor of the basic HLH transcription factors by forming heterodimers (1). Up-regulation of Id-1 is frequently observed in numerous types of human cancer, such as lung (2) and prostate cancer (3). Research on bladder cancer has also shown that the up-regulation of Id-1 is associated with clinical staging, invasive ability and increased epidermal growth factor receptor (EGFR) expression in bladder cancer cells (4). Recently, we found that regulation of Id-1 had an effect on the chemosensitivity of bladder cancer cells: overexpression of Id-1 decreased sensitivity to epirubicin and suppressed epirubicin-induced apoptosis, while the down-regulation of Id-1 increased chemosensitivity and epirubicin-induced apoptosis (5). These results indicate that Id-1 may play a key role in tumorigenesis and cancer development, and may thus be a potential target in the treatment of bladder cancer.

In recent years, increasing emphasis has been placed on identifying naturally occurring anticancer substances that inhibit, retard or reverse the process of carcinogenesis. Garlic (Allium sativum) is a herbal vegetable that has been widely used for thousands of years. Experimental and epidemiological studies have indicated that increased consumption of garlic results in a significantly decreased risk of mammary (6), esophageal (7), colon (8) and lung (9) cancer. Several mechanisms have been proposed to explain the cancer preventive effects of Allium vegetables. These include the inhibition of mutagenesis through metabolism inhibition, the inhibition of DNA adduct formation, free-radical scavenging and antiproliferating activities, and the induction of apoptosis (10). Several individual compounds have been isolated from garlic; certain of these have been found to have an active anticancer effect. S-allylmercaptocysteine (SAMC), a water-soluble organosulfur compound derived from ethanol extracts of garlic, has been demonstrated to have a potent therapeutic effect on human cancer cells. Studies in vivo and in vitro have shown that SAMC is not only able to directly inhibit the proliferation of a variety of cancer cell lines (11), but also to 
suppress cancer growth in animal models (12). These findings suggest that SAMC may be an effective agent in the treatment of primary human cancer.

In a previous study (5), we found that SAMC inhibits the proliferation of bladder cancer cells and induces their apoptosis. However, the pathway by which SAMC induces the apoptosis of human bladder cancer cells has yet to be determined. Therefore, this study aimed to investigate whether Id-1 expression modulates SAMC-induced apoptosis in human bladder cancer cells. To this end, we transfected a pBabe-Id-1 expression retroviral vector and retroviral vectors containing an Id-1-specific small interfering RNA oligonucleotide (si-Id-1) into two respective bladder cancer cell lines. We then examined whether regulation of Id-1 affects the sensitivity of these cells to SAMC. Our results suggest that the down-regulation of Id-1 increases sensitivity to SAMC-induced apoptosis, while the up-regulation of Id-1 in bladder cancer cells leads to decreased sensitivity to SAMC. Furthermore, the inactivation of Id-1 enhances the inhibitory effect of SAMC on the invasive and migratory abilities of bladder cancer cells.

\section{Materials and methods}

Cell lines and cell culture conditions. Two human bladder urothelial cancer cel1 lines, RT112 and MGH-U1, were obtained from the Department of Anatomy of the University of Hong Kong. The cells were maintained in RPMI-1640 medium (Sigma, St. Louis, MO, USA) supplemented with $5 \%(\mathrm{v} / \mathrm{v})$ fetal bovine serum, penicillin $(100 \mathrm{U} / \mathrm{ml})$ and streptomycin $(100 \mathrm{U} / \mathrm{ml})$ at $37^{\circ} \mathrm{C}, 5 \% \mathrm{CO}_{2}$. Cells were split and harvested using trypsin (Sigma), and drug exposures were performed in the cell incubator.

S-allylmercaptocysteine. SAMC (purity >95\%) was obtained from Wakunaga Pharmaceutical Co., Ltd. (Hiroshima, Japan). A stock solution of SAMC (5 mM) was freshly prepared in PBS according to the manufacturer's instructions.

Generation of stable Id-1 expressing transfectants. The pBabeId-1 expression retroviral vector and its corresponding vector control were used for the generation of stable transfectants in the RT112 cells. Briefly, the retroviral vector containing full length Id-1 cDNA (pBabe-Id-1) or pBabe-puro was transfected into the PG13 packaging cell line (obtained from ATCC) using the calcium phosphate method. After selection for 1 week in $4 \mu \mathrm{g} / \mathrm{ml}$ puromycin, the culture medium containing infectious viruses was harvested for retroviral infection of RT112 cells. Briefly, the virus-containing supernatant was mixed with an equal volume of fresh medium containing $8 \mu \mathrm{g} / \mathrm{ml}$ polybrene and then added to the RT112 cells. Puromycin $(1 \mu \mathrm{g} / \mathrm{ml})$, which killed all of the parental cells, was added $24 \mathrm{~h}$ later. Ten Id-1 stable transfectant clones were isolated 14 days after drug selection to generate RT112-pBabe-Id-1 clones. Vector control was generated from a pool of $>20$ individual clones transfected with pBabe.

Construction of si-RNA vectors and generation of stable si-Id-1 transfectants. The Id-1 si-RNA vector was generated using the GeneSuppressor System kit (Imgenex, San Diego,
CA, USA) according to the manufacturer's instructions. Vectors and transfection procedures were as described previously (5). The resulting vectors were transfected into the 293 packaging cell line using Fugene 6 reagent. Retroviruses were collected $48 \mathrm{~h}$ later, mixed with polybrene $(8 \mu \mathrm{g} / \mathrm{ml})$ and then incubated with MGH-U1 cells. Positive si-Id-1 clones were selected in neomycin $(600 \mu \mathrm{g} / \mathrm{ml})$ and a pool of stable transfectants was isolated after 6 days of drug selection.

Colony-forming assay. A single-cell suspension was prepared and seeded in 12-well plates at a density of 200 cells/well. Twenty-four hours after plating, the cells were treated with five concentrations of SAMC and incubated for 10-12 days, then fixed in $70 \%$ ethanol and stained with $1 \%$ (v/v) Giemsa blue (Merck, Damstadit, Germany). Colonies consisting of $>50$ cells were counted. Two wells were used for each concentration, and two wells treated with solvent only served as the controls. Colony forming ability after SAMC treatment was calculated as the ratio between the number of colonies in the treated wells and the untreated controls multiplied by $100 \%$. Each experiment was repeated at least three times.

3-(4,5-Dimethyl thiazol-2-yl)-2,5-diphenyl tetrazolium bromide (MTT) assay. Cell viability was measured using the MTT proliferation assay kit according to the experimental procedures described by the manufacturer (Boeringher, MO, USA). Briefly, 3,000 cells for RT112 and 1,500 cells for MGH-U1 were seeded in 96-well plates and cultured for $24 \mathrm{~h}$. Two concentrations of SAMC were added, $100 \mu \mathrm{M}$ for pBabe and Id-1, $150 \mu \mathrm{M}$ for sscon and si-Id-1, respectively. Cell viability was examined at different time point after treatment (24, 48, 72 and 96 h). Medium in the 72- and 96-h plates was changed at $48 \mathrm{~h}$ after treatment. Before testing, $20 \mu \mathrm{l}$ of MTT labeling reagent $(5 \mathrm{mg} / \mathrm{ml}$ MTT in PBS) was added and the cells were incubated for $4 \mathrm{~h}$ at $37^{\circ} \mathrm{C}$. Culture medium and MTT was then removed from the wells before the addition of $200 \mathrm{ml}$ dimethyl sulphoxide (DMSO) to each well. The plates were further incubated for $15 \mathrm{~min}$ at $37^{\circ} \mathrm{C}$ to dissolve the formazan crystals. Optical density was measured at a wavelength of $570 \mathrm{~nm}$ on a Labsystem multiscan microplate reader (Merck Eurolab, Dietikon, Switzerland). The MTT assay results are the optical density of the specimens recorded on a spectrophotometer, which reflects residual viable cell biomass.

4,6-Diamidino-2-phenylindole (DAPI) staining. Bladder cancer cells were seeded on 6 well-plates with cover slips. Cells were treated with SAMC at different doses for $24 \mathrm{~h}$. At the end of the incubation, cells were fixed in $4 \%$ paraformaldehyde for $15 \mathrm{~min}$. After washing twice for $5 \mathrm{~min}$ with $1 \mathrm{X}$ PBS, cells were incubated with DAPI $(0.5 \mu \mathrm{g} / \mathrm{ml})$ for $5 \mathrm{~min}$ at room temperature and then washed with $1 \mathrm{X}$ PBS for $5 \mathrm{~min}$. After washing, slides were mounted using Dako cytomation fluorescent mounting medium, and cell morphology was examined under a fluorescent microscopy. Cells which exhibited condensed chromatin at the periphery of the nuclear membrane or general fragmented morphology of nuclear bodies were considered to be undergoing apoptosis. A total of 500 cells were counted in five randomly selected fields per sample under a magnification of $\mathrm{x} 400$. The percentage of cells 
showing nuclear bodies was calculated as the ratio of number of apoptotic cells against the total cell number, counted and multiplied by $100 \%$.

Western blotting. Western blotting as carried out as previously described (13). In brief, whole-cell lysate was prepared by re-suspending cell pellets in lysis buffer, and protein concentrations were measured using the protein assay kit (Bio-Rad, Hercules, CA, USA). The protein suspension $(10-30 \mu \mathrm{g})$ was then loaded onto sodium dodecylsulfatepolyacrylamide gels for electrophoresis, then transferred to a polyvinylidene difluoride membrane (Amersham, Piscataway, NJ, USA). The membranes were incubated for $1-2 \mathrm{~h}$ at room temperature with primary antibodies against Id-1 (Santa Cruz Biotechnology, Santa Cruz, CA, USA), p27 Kip1 (BD Biosciences, San Diego, CA, USA), p21 ${ }^{\mathrm{WAF} 1 / \mathrm{CIP1}}$ (Dako, Tokyo, Japan), caspase-3, cleaved caspase-3, PARP, cleaved PARP, Bcl-2, Bax and $\beta$-actin (Cell Signaling Technology, Beverly, MA, USA), respectively. After washing with Tris-buffered saline Tween-20 (TBS-T), the membranes were incubated with a secondary antibody against rabbit, mouse or goat IgG. Signals were visualized using the ECL Western blotting system (Amersham).

Type I collagen invasion assay. A type I collagen invasion assay was carried out as described by Bracke et al (14) with minor modifications. Briefly, collagen solution was prepared by mixing all the pre-cooled $\left(4^{\circ} \mathrm{C}\right)$ components, including collagen type I, minimum essential medium (MEM), PBS, sodium bicarbonate $\left(\mathrm{NaHCO}_{3}\right)$ solution and sodium hydroxide $(\mathrm{NaOH})$. The solution was gently agitated to avoid the introduction of air bubbles. Mixture solution $(1.25 \mathrm{ml}$ per well) was added to the outer wells of a 6-well plate, while $1.35 \mathrm{ml}$ of the mixture solution was added to each of the middle wells. The collagen solution was allowed to solidify for $\sim 2 \mathrm{~h}$ at $37^{\circ} \mathrm{C}$ in a cell culture incubator. Subsequently, cells were trypsinized and resuspended in culture medium for a total volume of $2 \mathrm{ml}$. The cell suspension was then poured onto the solidified gel and allowed to attach and grow on the gel for $12 \mathrm{~h}$. Twelve hours later, the culture medium was replaced with low concentration SAMC medium $(100 \mu \mathrm{M})$. Cell morphology was observed $24 \mathrm{~h}$ later under an inverted microscope. Solitary cells with rounded shape were considered non-invading cells, while cells with cytoplasmic extension, an indicator of invasive behavior, were considered invading cells. Ten random microscopic fields at a magnification of $x 400$ were counted for each well. The percentage of invading cells was calculated as number of invading cells/ total number of cells x $100 \%$.

Wound closure assay. Bladder cancer cells $\left(4 \times 10^{5}\right)$ were seeded into 6-well culture plates and allowed to grow to $90-95 \%$ confluence in medium for $24 \mathrm{~h}$. Similar-sized wounds were introduced to monolayer cells using a sterile yellow pipette tip, and medium with floating cells was carefully removed. Pre-warmed medium $(2 \mathrm{ml})$ with low dose of SAMC $(100 \mu \mathrm{M})$ was added. Images were captured at a magnification of x100 using a phase contrast microscope immediately after wound induction at 48- and 96-h intervals for Id-1 transfectants, and at 12- and 24-h intervals for si-Id-1 transfectants.
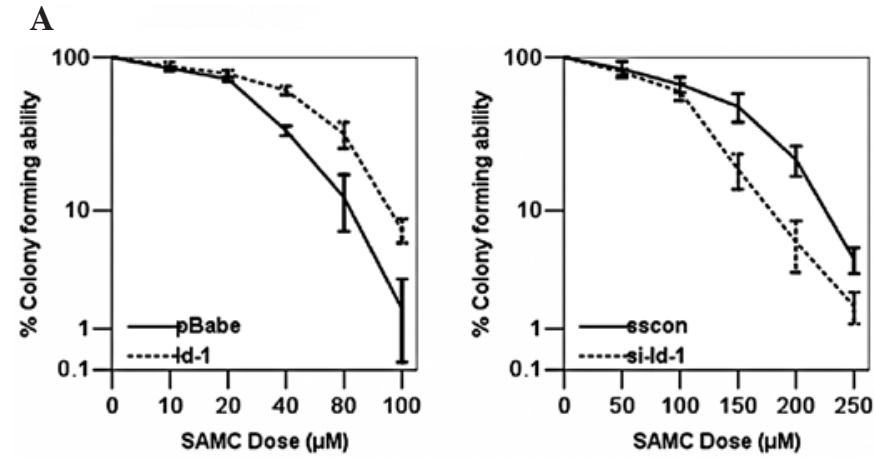

B
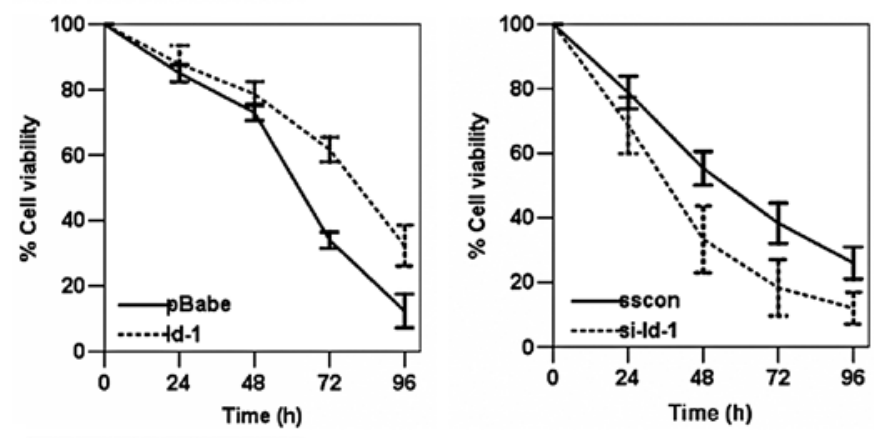

C

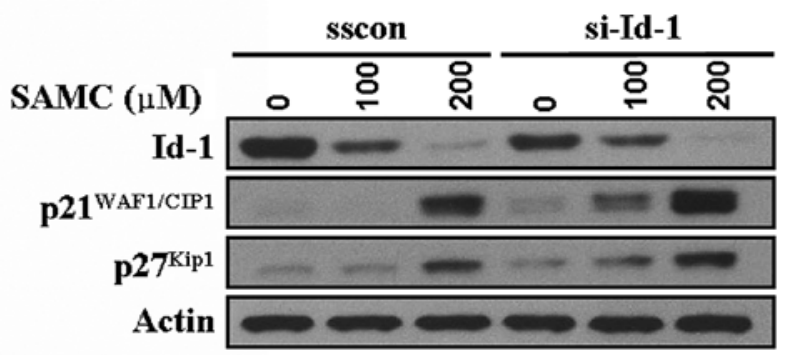

Figure 1. Cellular sensitivity to SAMC mediated by Id-1 in bladder cancer cells. (A) Colony forming ability of SAMC-treated cell lines. Colony formation assays were performed in pBabe, Id-1, sscon and si-Id-1 clones after exposure to six doses of SAMC. Cell lines with relatively high levels of Id-1 (Id-1 and sscon) were more resistant to SAMC than those with relatively low levels of Id-1 (pBabe and si-Id-1). Survival curves are based on the means and standard deviation (SD) of the results of three separate experiments. (B) MTT assay of cell viability after exposure to identical doses of SAMC at different time points. Cell viability was higher in the Id-1 and sscon clones than in the pBabe and si-Id-1 clones, respectively. The data represent the optical density ratio between the treated and untreated cells at the different time points. (C) Expression of Id-1, p21 $1^{\mathrm{WAF} 1 / \mathrm{CIP1}}$ and $\mathrm{p} 27^{\mathrm{Kip} 1}$ protein in SAMCtreated cell lines. The results of Western blotting show that the expression levels of Id-1 were decreased and the expression levels of $\mathrm{p} 21^{\mathrm{WAF} / \mathrm{CIP} 1}$ and p27 ${ }^{\text {Kipl }}$ were increased after treatment with increasing doses of SAMC. The expression levels of $\mathrm{p} 21^{\mathrm{WAFI} / \mathrm{CIP} 1}$ and $\mathrm{p} 27^{\mathrm{Kip} 1}$ were much higher in the si-Id-1 clones than in the vector control clones.

\section{Results}

Effect of Id-1 on cell sensitivity to SAMC. The results of the generation of stable transfectants have been described previously (5). Briefly, the bladder cancer cell line RT112 was shown to express a relatively low level of Id-1. Therefore, pBabe-Id-1 expression retroviral vector was transfected into an RT112 cell line, and two stably transfected clones were generated. The MGH-U1 bladder cancer cell line was shown to express a relatively high level of Id-1. Therefore, a retroviral vector 


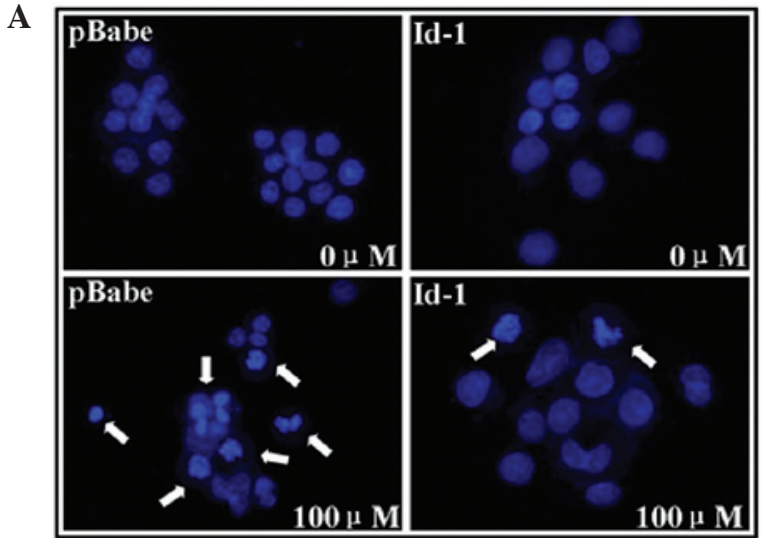

C

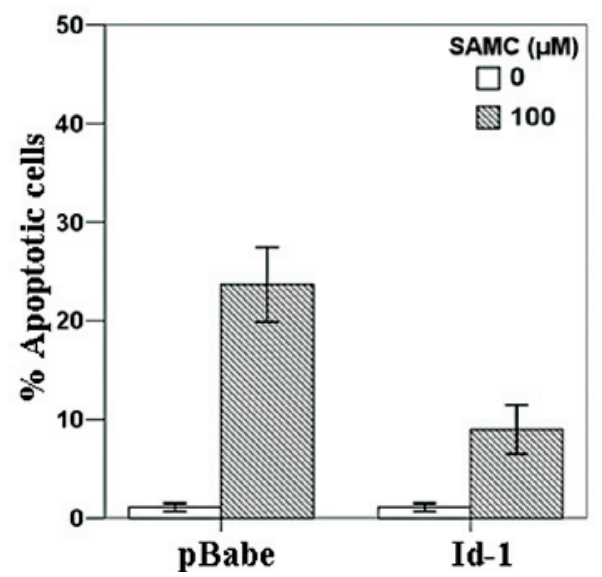

B

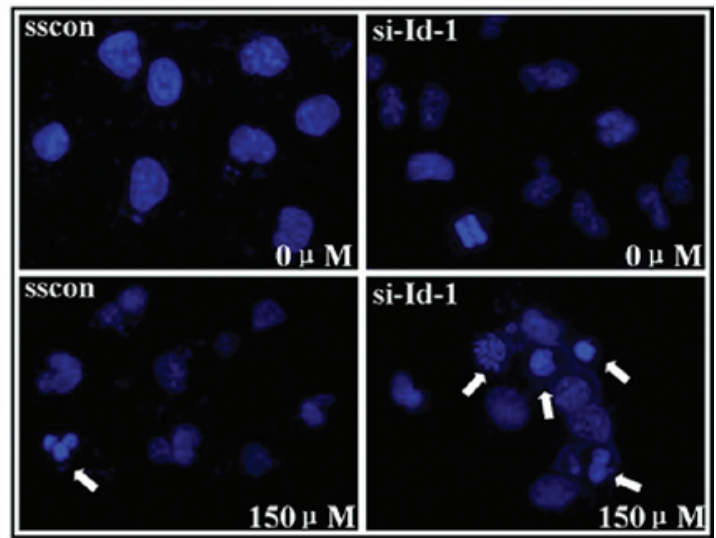

D

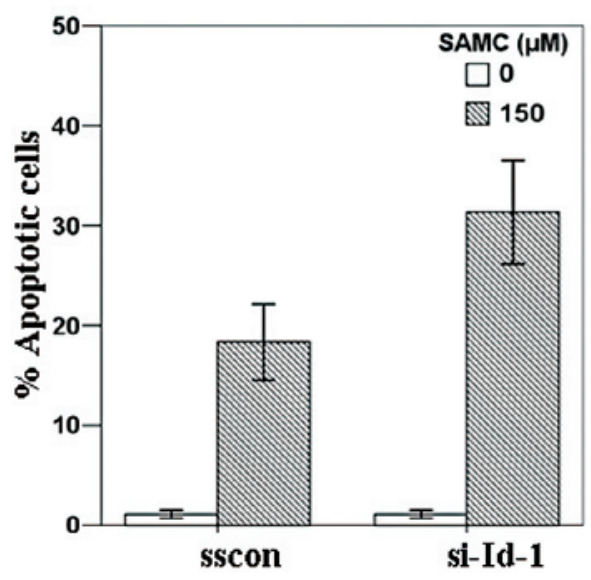

Figure 2. Effect of Id-1 regulation on SAMC-induced apoptosis. (A and B) Representative images of apoptotic cells by DAPI staining. Cells with condensed chromatin or fragmented nuclei were considered to be undergoing apoptosis (arrows). (C and D) Mean rate of apoptosis in the different transfectants and vector controls after exposure to SAMC. The rate of apoptosis was lower in the Id-1 and sscon transfectants than in the pBabe and si-Id-1 transfectants. Error bars indicate the standard deviation.

containing a siRNA oligonucleotide targeted to the Id-1 gene was transfected into the MGH-U1 cell line, and one stably transfected clone was generated. The selected transfected clones and their corresponding vector control clones were used in the present study to perform the related experiments.

To investigate the effect of Id-1 regulation on the survival of cells treated with SAMC, a colony formation assay was performed on the two pairs of transfectants. As shown in Fig. 1A, the cancer cells were treated with six doses of SAMC, and SAMC was found to inhibit the clonogenesity of both pairs of cell lines in a dose-dependent manner. Moreover, the colony forming ability of the pBabe clone was much lower than that of the Id-1 clone, while that of the sscon clone was much higher than that of the si-Id-1 clone, after exposure to identical doses of SAMC. These results indicate that decreased Id-1 expression is associated with decreased cell survival in bladder cancer cells treated with SAMC, while the up-regulation of Id-1 has the reverse effect.

Next, the MTT assay was used to investigate whether Id-1 inactivation is capable of increasing the sensitivity of bladder cancer cells to SAMC by reducing cell viability. Cells were treated with SAMC at relatively low concentrations $(100 \mu \mathrm{M}$ for $\mathrm{pBabe}$ and Id-1, $150 \mu \mathrm{M}$ for sscon and si-Id-1) designed to induce slow cell death over a relatively long period (72 and $96 \mathrm{~h}$ ), since previous studies on bladder cancer cells showed that treatment with relatively high doses of SAMC $(\geq 200 \mu \mathrm{M})$ leads to the rapid induction of cell death. Fig. 1B shows that treatment with low concentrations of SAMC induced a loss in cell viability in the bladder cancer cells in a time-dependent manner. In addition, cell viability was higher in the Id-1 and sscon clones than in the pBabe and si-Id-1 clones, respectively, after exposure to identical doses of SAMC at different time points.

To investigate the underlying mechanisms responsible for the increased sensitivity to SAMC-induced growth arrest in the si-Id-1 clones, the expression levels of Id-1, p21 $1^{\mathrm{WAF} 1 / \mathrm{CIP} 1}$ and p2 $7^{\mathrm{Kip} 1}$ protein were investigated by Western blotting $(15,16)$. Fig. 1C shows that after treatment with increased doses of SAMC $(0,100$ and $200 \mu \mathrm{M})$, the expression levels of Id-1 were decreased while the expression levels of $\mathrm{p} 21^{\mathrm{WAF} 1 / \mathrm{CIP} 1}$ and p2 $7^{\mathrm{Kip} 1}$ were increased. Furthermore, the expression levels of $\mathrm{p} 21^{\mathrm{WAF} 1 / \mathrm{CIP} 1}$ and $\mathrm{p} 27^{\mathrm{Kipl}}$ were much higher in the si-Id-1 clones than in the vector control clones. Taken together, these results indicate that the inactivation of Id-1 might increase the susceptibility of bladder cancer cells to SAMC-induced cell death.

Effect of Id-1 regulation on SAMC-induced apoptosis. Previous studies have shown that Id-1 functions to protect cancer cells against apoptosis (17). In this study, to determine whether the increased sensitivity to SAMC in the si-Id-1 transfectants was due to an increased sensitivity to apoptosis, DAPI staining and Western blot analysis of apoptotic-related 
A

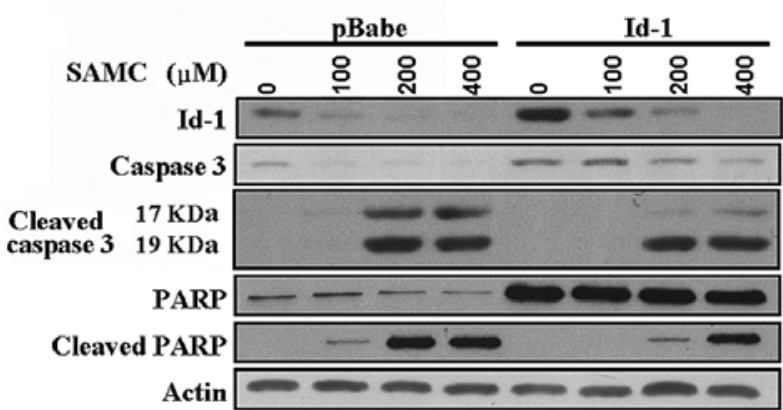

B

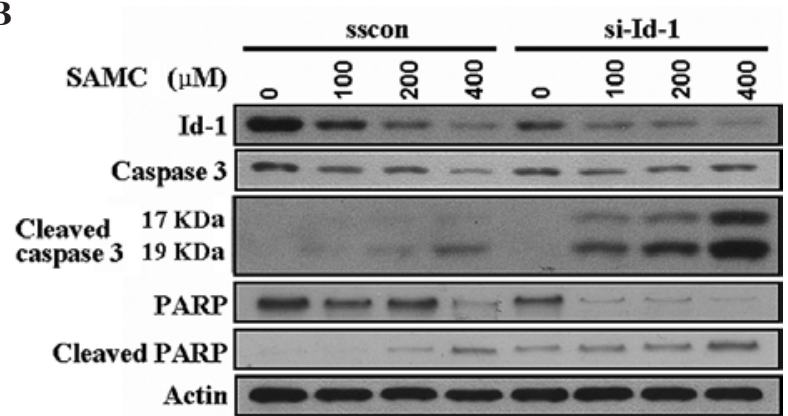

Figure 3. Expression of apoptosis-related proteins (A, pBabe and Id-1; B, sscon and si-Id-1) analyzed by Western blotting. The apoptotic pathway activated by SAMC in bladder cancer cells was mediated by Id-1 protein. The expression of cleaved caspase- 3 and cleaved PARP increased with an increase in the dose of SAMC, and decreased with an increase in the expression level of Id-1.

proteins were performed. Cells were stained by DAPI and cell morphology was observed using fluorescence microscopy. Fig. 2A and B show the results of DAPI staining of the bladder cancer cells after SAMC treatment. Cells with condensed chromatin or fragmented nuclei were considered apoptotic. Apoptotic nuclear bodies were observed in the cell lines treated with SAMC at concentrations of 100 or 150 $\mu \mathrm{M}$. Fig. 2C and D illustrate the percentage of apoptotic cells induced by SAMC. In the Id-1 clones, $9 \%$ of cells underwent apoptosis after treatment with $100 \mu \mathrm{M}$ of SAMC, which was significantly lower than in the control $(23.67 \%)(\mathrm{p}<0.05)$. In the si-Id-1 clones, $31.33 \%$ of cells treated with SAMC at $150 \mu \mathrm{M}$ were in the process of undergoing apoptosis, which was significantly higher than in the vector control $(18.33 \%)$ $(p<0.05)$. These results demonstrate that SAMC is capable of inducing morphological changes associated with apoptosis in the nuclei of bladder cancer cells, and that this effect is enhanced by the inactivation of Id-1.

Since nuclear apoptotic bodies were demonstrated by DAPI, the expression of apoptosis-related proteins was analyzed by Western blotting to determine whether these proteins are associated with apoptosis induced by SAMC. As demonstrated in Fig. 3, after exposure to increasing doses of SAMC $(0,100,200$ and $400 \mu \mathrm{M})$, total caspase-3 was cleaved into two fragments of 17 and $19 \mathrm{kDa}$. Expression of cleaved caspase-3 increased with an increase in the dose of SAMC and decreased with an increase in the expression level of Id-1. By contrast, the expression levels of corresponding total caspase-3 decreased with an increase in the dose of SAMC and increased with an increase in the expression level of Id-1. In addition, full length PARP, which is a substrate of caspase-3, was found to be cleaved into an $85-\mathrm{kDa}$ fragment after treatment with SAMC. Changes in full-length and cleaved PARP were similar to those observed with total and cleaved caspase-3, respectively. These results further support the negative role of Id-1 in SAMC-induced apoptosis, and suggest that inactivation of Id-1 may induce increased sensitivity to SAMC in bladder cancer cells.

Effect of Id-1 inactivation on the SAMC-induced inhibition of cell invasion and migration. One of the characteristics of malignant cancer cells is their inclination to invade areas outside their usual territory (18). Invading cancer cells infiltrate into adjacent tissues by disrupting tissue architecture and penetrating the basement membrane and extracellular matrix (19). To determine whether the inactivation of Id-1 is associated with the inhibition of bladder cancer cell invasion in response to SAMC, a type I collagen invasion assay was performed. As shown in Fig. 4A, after transfection, a lower percentage of si-Id-1 cells compared to sscon cells was observed in the semisolid collagen gel, and si-Id-1 cells displayed an elongated or scattered morphology, indicating their weakened ability to invade the extracellular matrix (32.7 vs. $45.1 \%$, p<0.05). When the cells were treated with $100 \mu \mathrm{M} \mathrm{SAMC}$, the percentage of colonies showing elongated morphology was reduced to $24.4 \%$ for sscon and $14.3 \%$ for si-Id-1 cells $(\mathrm{p}<0.05)$. These results suggest that inactivation of Id-1 effectively increases the inhibitory effect of SAMC on bladder cancer cell invasion.

Increased migration rate is one of the key factors responsible for cancer metastasis (20). To determine whether Id-1 had any effect on the migration ability of bladder cancer cells treated with SAMC, a wound closure assay was performed. As shown in Fig. 4B, similar-sized wounds were introduced in monolayer cells, and the speed of wound closure was monitored. In the Id-1 cells untreated by SAMC, the wound gap was gradually filled by migrating cells, and was almost closed by $96 \mathrm{~h}$ after wound induction, while a wide gap remained in the pBabe cells untreated by SAMC. By contrast, after exposure to SAMC $(100 \mu \mathrm{M})$, the wounds of Id-1 cells were still open after $96 \mathrm{~h}$, and the speed of wound closure in pBabe cells was greatly reduced. Similar results were observed in sscon and si-Id-1 cells, though at shorter experimental time points (Fig. 4C). As the speed of wound closure reflects the migration ability of cancer cells, these results indicate that SAMC treatment inhibits cell migration, and that bladder cancer cells with inactivated Id-1 have increased sensitivity to the inhibitory effect of SAMC on migration.

\section{Discussion}

Immunotherapy with BCG for high-risk non-muscle invasive bladder cancer is effective; however, BCG is associated with marked toxicity, which limits its widespread use. In addition, in cases of advanced bladder cancer, the effect of cisplatincontaining systematic polychemotherapy is limited and has apparent side-effects. Therefore, the identification of safe and effective alternative treatments is desirable. In recent 


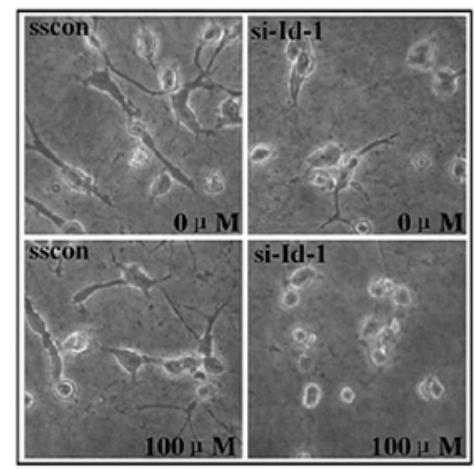

B

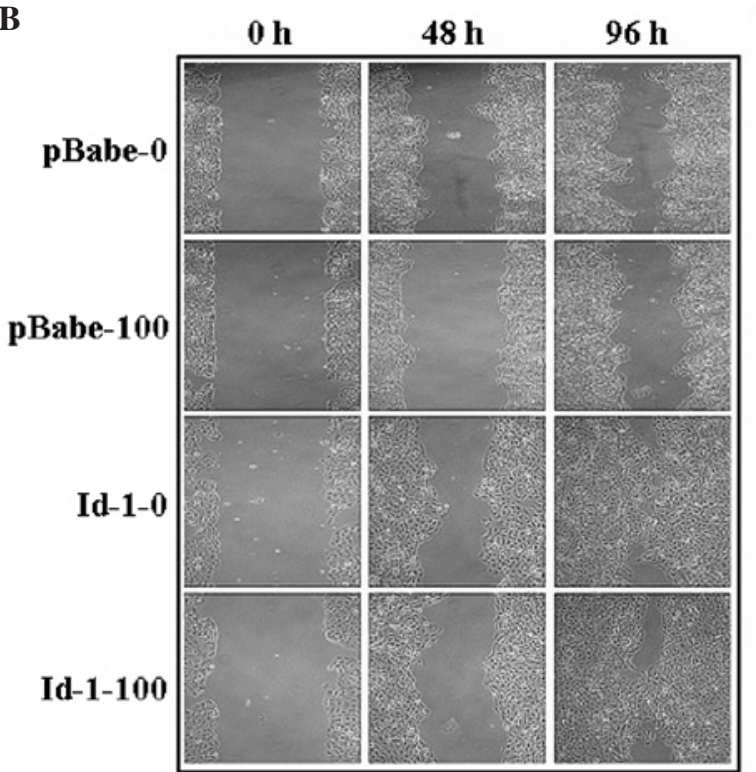

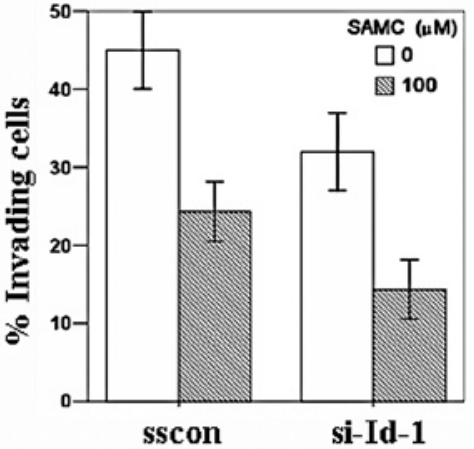

C<smiles>[Mg]=[Mg]</smiles>

$\mathbf{0 h}$ $12 \mathrm{~h}$ $24 \mathrm{~h}$

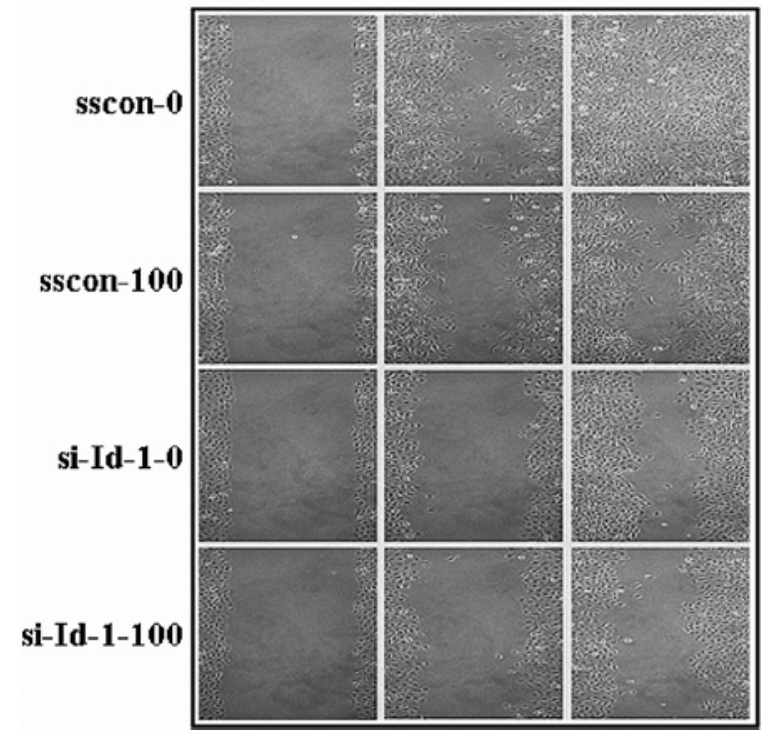

Figure 4. Effect of Id-1 regulation on the invasion and migration ability of SAMC-treated bladder cancer cells. (A) Collagen invasion assay showing the morphological appearance of the SAMC-treated sscon and si-Id-1 cell lines (left panels) compared to the percentage of invading cells (right panels). Silencing of Id-1 caused an increase in the inhibitory effect of SAMC on bladder cancer cell invasion. (B) Comparison of wound closure ability between pBabe and Id-1 cell lines treated with SAMC. The speed of wound closure was slower after SAMC treatment in both cell lines, and the speed of pBabe wound closure was greatly reduced. (C) Comparison of wound closure ability between sscon and si-Id-1 cell lines treated with SAMC. The speed of wound closure was slower after SAMC treatment in both cell lines and the speed of si-Id-1 wound closure was greatly reduced. Data are presented as the mean \pm SD. Representative images are shown.

years, increasing emphasis has been placed on identifying naturally occurring anticancer substances due to their low toxicity. Numerous studies have been conducted to explore the mechanisms of action of these substances and to improve their effectiveness. The present study focused on SAMC, a water-soluble garlic derivative, and demonstrated that it exerts an anticancer effect by inhibiting cell proliferation, apoptosis, invasion and migration, which are all mediated by Id-1 in bladder cancer cells.

Two human bladder urothelial cancer cell lines, RT112 and $\mathrm{MGH}-\mathrm{U} 1$, were used as in vitro models to test the regulatory effect of Id-1 on SAMC-induced cell death. It was demonstrated that SAMC at the appropriate concentrations inhibited the colony forming ability of bladder cancer cells (Fig. 1A). Cell lines with high Id-1 expression (Id-1 and sscon) were more resistant to SAMC. By contrast, cell lines with relatively lower Id-1 expression (pBabe and si-Id-1) had increased sensitivity to SAMC. The evaluation of cell viability by MTT assay also showed that SAMC had an inhibitory effect on cell growth in a time-dependent manner, and that this effect was regulated by
Id-1 (Fig. 1B). The inhibitory effect of SAMC and Id-1 downregulation on bladder cancer cells was also confirmed by the detection of up-regulated expression levels of p21WAF1/CIP1 and p27Kip1 protein (Fig. 1C). p21WAF1/CIP1 and p27Kip1 are cell cycle regulatory proteins that negatively mediate cell cycle progression (21). Previous reports have suggested that Id-1 inhibits the E2A (product of adenovirus early region $2 \mathrm{~A}$ gene)-dependent expression of p21WAF1/CIP1 (22), and that ectopic expression of Id-1 is associated with the down-regulation of p27Kip1 (23). In this study, we observed that almost all SAMC-treated si-Id-1 clones had higher p21WAF1/CIP1 and $\mathrm{p} 27 \mathrm{Kip} 1$ expression levels compared to the corresponding SAMC-treated sscon clones, and that this increase in expression was dose-dependent. This indicates that SAMC treatment and the suppression of Id-1 lead to increased p21WAF1/ CIP1 and p27Kip1 expression. SAMC treatment reduced Id-1 expression levels, indicating that Id-1 may be one of the downstream effectors for SAMC-induced growth arrest.

The ultimate role of an anticancer agent is to inhibit cellular proliferation in order to induce the apoptosis of cancer 
cells (24). Apoptosis is a process involving programmed cell death, and its mechanisms have been the focus of numerous studies, along with the identification of compounds that influence apoptosis. During apoptosis, chromatin in the nucleus is condensed and endo-nuclease activation results in DNA fragmentation. The resulting DNA fragmentation is marginalized to the nuclear periphery, leading to the formation of apoptotic bodies (25). In the present study, in order to further investigate the anticancer effect of SAMC and the regulatory effect of Id-1 on bladder cancer cells, several assays were performed to assess SAMC-induced apoptosis. The results of DAPI staining demonstrated that incubation with relatively low concentrations SAMC induced nuclear apoptotic bodies in bladder cancer cells, and that more extensive DNA fragmentation was induced in clones with lower expression of Id-1 (Fig. 2). To further confirm that the apoptotic pathway activated by SAMC in bladder cancer cells was mediated by Id-1 protein, the expression levels of several apoptosis-related proteins were investigated by Western blotting. Caspases including caspase-3, $-6,-7,-8$ and -9 are present in precursor forms and are cleaved into smaller molecular weight subunits after apoptotic activation (26). The activation of caspases plays an essential role in apoptosis. Caspase- 3 is the key mediator of the final stages of apoptosis; activation of caspase-3 leads to the cleavage of its substrate, poly (ADP ribose) polymerase (PARP) (27), which leads to DNA fragmentation and oligonucleosome formation. The results of this study revealed that caspase-3 was cleaved into its subunits in a dose-dependent manner after SAMC treatment (Fig. 3), and that the expression of precursor caspase-3 was similarly decreased. Decreased levels of Id- 1 in the pBabe and si-Id-1 compared to the Id-1 and sscon transfectants were correlated with higher levels of cleaved caspase-3. In combination, these results suggest that SAMC may induce apoptosis in bladder cancer cells in a caspase-3-dependent manner, and that this may be mediated by Id-1 expression.

Tumor progression involves the invasion of cancer cells into adjacent regions or migration to distal regions, which leads to the metastasis of the original tumor. In addition to having a suppressive effect on tumor growth, SAMC has previously been reported to prevent metastasis in human cancer cells (28). Id-1 protein expression has also been found to be correlated with disease progression in several types of cancer (29), and screening of breast cancer cell lines revealed that the expression of Id-1 was directly correlated with the invasiveness of these cell lines (30). The current findings indicate that a relatively low dose of SAMC is capable of suppressing the invasion (Fig. 4A) and migration (Fig. 4B) of bladder cancer cells. In addition, the inhibitory effect of SAMC on bladder cancer cell invasion and migration was increased in cells with inactivated Id-1 (Fig. 4C). These results suggest that SAMC may suppress the invasion and migration abilities of bladder cancer cells through inactivation of Id-1.

In conclusion, our results suggest that the down-regulation of Id-1 results in an increase in the SAMC-induced apoptosis of bladder cancer cells. The inhibitory effect of SAMC on cell proliferation, invasion and migration is also increased by Id-1 inactivation. These findings may lead to the development of novel therapeutic strategies for the treatment of bladder cancer.

\section{Acknowledgements}

This study was supported by the Peking University People's Hospital Research and Development Fund to Dr Ke Xin $\mathrm{Xu}$ (RDB2008-30).

\section{References}

1. Benezra R, Davis RL, Lockshon D, Turner DL and Weintraub H: The protein Id: a negative regulator of helix-loop-helix DNA binding proteins. Cell 61: 49-59, 1990.

2. Kamalian L, Gosney JR, Forootan SS, Foster CS, Bao ZZ, Beesley $\mathrm{C}$ and $\mathrm{Ke} \mathrm{Y}$ : Increased expression of Id family proteins in small cell lung cancer and its prognostic significance. Clin Cancer Res 14: 2318-2325, 2008

3. Ouyang XS, Wang X, Lee DT, Tsao SW and Wong YC: Overexpression of ID-1 in prostate cancer. J Urol 167: 2598-2602, 2002.

4. Ding Y, Wang G, Ling MT, et al: Significance of Id-1 upregulation and its association with EGFR in bladder cancer cell invasion. Int J Oncol 28: 847-854, 2006.

5. Hu H, Han HY, Wang YL, et al: The role of Id-1 in chemosensitivity and epirubicin-induced apoptosis in bladder cancer cells. Oncol Rep 21: 1053-1059, 2009.

6. Schaffer EM, Liu JZ, Green J, Dangler CA and Milner JA: Garlic and associated allyl sulfur components inhibit N-methyl$\mathrm{N}$-nitrosourea induced rat mammary carcinogenesis. Cancer Lett 102: 199-204, 1996.

7. Yu FS, Yu CS, Lin JP, Chen SC, Lai WW and Chung JG: Diallyl disulfide inhibits $\mathrm{N}$-acetyltransferase activity and gene expression in human esophagus epidermoid carcinoma CE 81T/VGH cells. Food Chem Toxicol 43: 1029-1036, 2005.

8. Hosono T, Fukao T, Ogihara J, Ito Y, Shiba H, Seki T and Ariga T: Diallyl trisulfide suppresses the proliferation and induces apoptosis of human colon cancer cells through oxidative modification of beta-tubulin. J Biol Chem 280: 41487-41493, 2005.

9. Wu XJ, Kassie F and Mersch-Sundermann V: The role of reactive oxygen species (ROS) production on diallyl disulfide (DADS) induced apoptosis and cell cycle arrest in human A549 lung carcinoma cells. Mutat Res 579: 115-124, 2005.

10. Shukla Y and Kalra N: Cancer chemoprevention with garlic and its constituents. Cancer Lett 247: 167-181, 2007.

11. Xiao D, Pinto JT, Soh JW, et al: Induction of apoptosis by the garlic-derived compound S-allylmercaptocysteine (SAMC) is associated with microtubule depolymerization and c-Jun $\mathrm{NH}(2)-$ terminal kinase 1 activation. Cancer Res 63: 6825-6837, 2003.

12. Chu Q, Lee DT, Tsao SW, Wang X and Wong YC: S-allylcysteine, a water-soluble garlic derivative, suppresses the growth of a human androgen-independent prostate cancer xenograft, CWR22R, under in vivo conditions. BJU Int 99: 925-932, 2007.

13. Ouyang XS, Wang X, Ling MT, Wong HL, Tsao SW and Wong YC: Id-1 stimulates serum-independent prostate cancer cell proliferation through inactivation of p16(INK4a)/pRB pathway. Carcinogenesis 23: 721-725, 2002.

14. Bracke ME, Boterberg T, Bruyneel EA and Mareel MM: Collagen invasion assay. In: Metastasis Research Protocols. Brooks SA and Schumacher U (eds). Humana Press, Totowa, NJ, pp81-89, 2001.

15. Datto MB, Li Y, Panus JF, Howe DJ, Xiong Y and Wang XF: Transforming growth factor beta induces the cyclin-dependent kinase inhibitor p21 through a p53-independent mechanism. Proc Natl Acad Sci USA 92: 5545-5549, 1995.

16. Polyak K, Kato JY, Solomon MJ, Sherr CJ, Massague J, Roberts JM and Koff A: P27Kip1, a cyclin-Cdk inhibitor, links transforming growth factor-beta and contact inhibition to cell cycle arrest. Genes Dev 8: 9-22, 1994.

17. Cheung HW, Ling MT, Tsao SW, Wong YC and Wang X: Id-1-induced Raf/MEK pathway activation is essential for its protective role against taxol-induced apoptosis in nasopharyngeal carcinoma cells. Carcinogenesis 25: 881-887, 2001.

18. Compagni A and Christofori G: Recent advances in research on multistage tumorigenesis. Br J Cancer 83: 1-5, 2000.

19. Guo W and Giancotti FG: Integrin signalling during tumour progression. Nat Rev Mol Cell Biol 5: 816-826, 2004.

20. Mareel M and Leroy A: Clinical, cellular, and molecular aspects of cancer invasion. Physiol Rev 83: 337-376, 2003. 
21. Morgan DO: Principles of CDK regulation. Nature 374: 131-134, 1995.

22. Prabhu S, Ignatova A, Park ST and Sun XH: Regulation of the expression of cyclin-dependent kinase inhibitor p21 by E2A and Id proteins. Mol Cell Biol 17: 5888-5896, 1997.

23. Everly DN Jr, Mainou BA and Raab-Traub N: Induction of Id1 and Id 3 by latent membrane protein 1 of Epstein-Barr virus and regulation of $\mathrm{p} 27 / \mathrm{Kip}$ and cyclin-dependent kinase 2 in rodent fibroblast transformation. J Virol 78: 13470-13478, 2004.

24. Westin P, Stattin P, Damber JE and Bergh A: Castration therapy rapidly induces apoptosis in a minority and decreases cell proliferation in a majority of human prostatic tumors. Am J Pathol 146: $1368-1375,1995$

25. Lee EC and Tenniswood M: Programmed cell death and survival pathways in prostate cancer cells. Arch Androl 50: 27-32, 2004.

26. Stennicke HR and Salvesen GS: Properties of the caspases. Biochim Biophys Acta 1387: 17-31, 1998.
27. Tewari M, Quan LT, O'Rourke K, et al: Yama/CPP32 beta, a mammalian homolog of CED-3, is a CrmA-inhibitable protease that cleaves the death substrate poly(ADP-ribose) polymerase. Cell 81: 801-809, 1995.

28. Chu Q, Ling MT, Feng H, Cheung HW, Tsao SW, Wang X and Wong YC: A novel anticancer effect of garlic derivatives: inhibition of cancer cell invasion through restoration of E-cadherin expression. Carcinogenesis 27: 2180-2189, 2006.

29. Schindl M, Oberhuber G, Obermair A, Schoppmann SF, Karner B and Birner P: Overexpression of Id-1 protein is a marker for unfavorable prognosis in early-stage cervical cancer. Cancer Res 61: 5703-5706, 2001.

30. Desprez PY, Lin CQ, Thomasset N, Sympson CJ, Bissell MJ and Campisi J: A novel pathway for mammary epithelial cell invasion induced by the helix-loop-helix protein Id-1. Mol Cell Biol 18: 4577-4588, 1998. 